\title{
EPIDIDYMAL FUNCTION IN THE VASECTOMIZED RABBIT
}

\author{
R. JONES* \\ Unit of Reproductive Biology, Life Sciences, Liverpool University, \\ PO Box 147, Liverpool L69 3BX
}

(Received 6th Fuly 1973)

As yet, there is little knowledge as to the possible side effects of vasectomy on the function of the epididymis. In rats and bulls, it has been reported that vasectomy causes a spermatocoele to develop at the site of ligation on the vas deferens or in the epididymis (Amann \& Almquist, 1962; Igboeli \& Rakha, 1970; Hooker \& Gilmore, 1972); in other species, notably the rabbit, this does not occur (Macmillan, Desjardins, Kirton \& Hafs, 1968; Paufler \& Foote, 1969). Some reports have claimed that vasectomy causes atrophy of the testis and disruption of spermatogenesis (Laumas \& Uniyal, 1967; Sacher \& Schilling, 1973) but most workers agree that, at least in the early stages, vasectomy only affects the terminal parts of the epididymis. It is not known what influence this might have on the normal maturation and survival of spermatozoa in the epididymis, a question which arises when an attempt is made to reverse the operation and restore normal fertility.

Recently, Jones \& Glover (1973a, b) investigated the chemical composition of the luminal plasma from the cauda epididymidis of normal and castrated rabbits. They concluded that the epididymis actively maintains a stable 'milieu' in the lumen of the duct by its capacity to absorb and secrete, and that the plasma reflects the function of the epididymis and that of the contained spermatozoa. The purpose of the present study was to investigate the effects of vasectomy on the function of the epididymis by examining the chemical composition of the luminal plasma.

Twenty-four adult male rabbits were anaesthetized with an intravenous injection of sodium pentobarbitone and bilaterally vasectomized through a scrotal incision. The ductus deferens was ligated 5 to $6 \mathrm{~cm}$ from the cauda epididymidis but was not severed. Recovery was uneventful and the luminal contents were collected from the cauda epididymidis after 2, 6, 12 and 24 weeks by the cannulation procedure of Jones \& Glover (1973a). The epididymal contents were centrifuged at $12,000 \mathrm{~g}$ for $5 \mathrm{~min}$, the spermatocrit was measured and the supernatant plasma was analysed for sodium, potassium, magnesium, and chloride ions, total protein, glycerylphosphorylcholine (GPC), inorganic phosphate, osmotic pressure, $\mathrm{pH}$, alkaline phosphatase, acid phosphatase, $\beta$-N-acetylglucosaminidase and lactic dehydrogenase (LDH), as described by

* Present address: A.R.G. Unit of Reproductive Physiology and Biochemistry, 307 Huntingdon Road, Cambridge CB3 0JQ. 
Jones \& Glover (1973a). In some instances, specimens were fixed in Bouin's fluid and spermatozoa stained with nigrosin-eosin (Campbell, Dott \& Glover, 1956).

There was a considerable increase in the size of the cauda epididymidis 6 weeks after vasectomy and by 24 weeks the terminal regions of the epididymis and ductus deferens were so distended with spermatozoa and fluid that they were larger than the testis (Pl. 1, Figs. 1, 2 and 3). At this time, 2 to $3 \mathrm{ml}$ of luminal contents could be collected whereas it is difficult to obtain more than $100 \mu \mathrm{l}$ from intact animals. Spermatocoeles did not develop at the site of

Table 1. The chemical composition of epididymal plasma from the cauda epididymidis of rabbits at different stages after vasectomy compared to that of intact controls

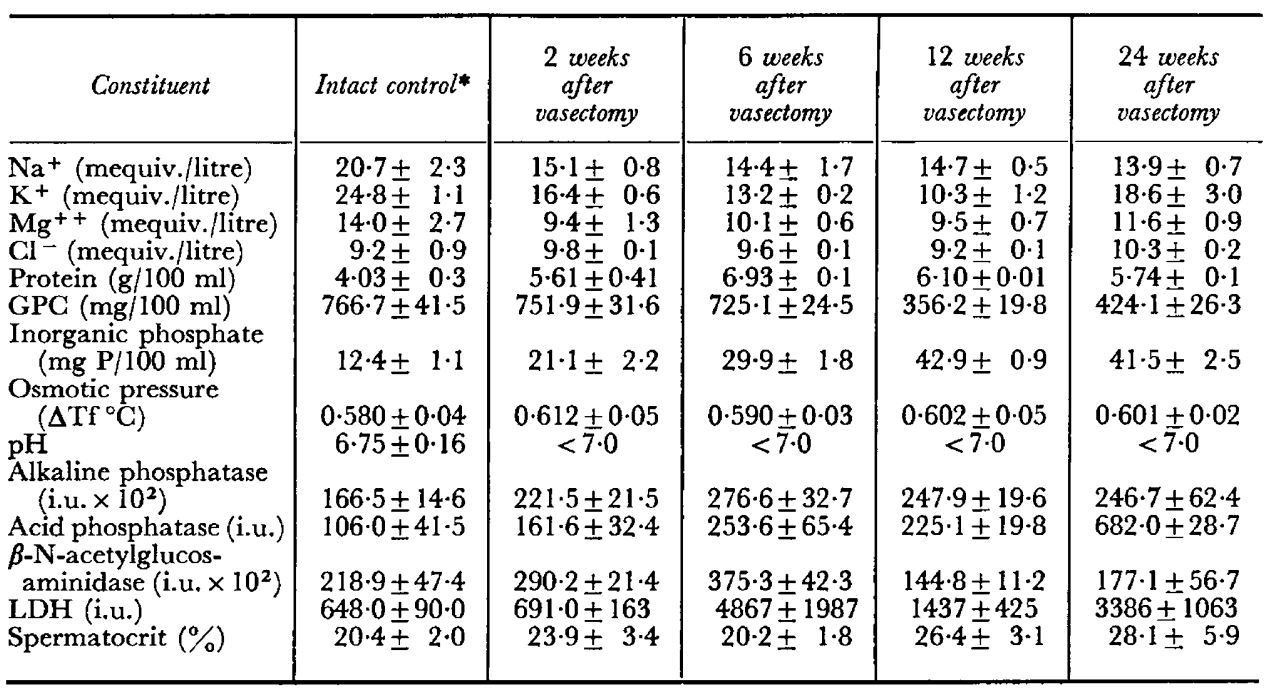

Each value is the mean for three animals \pm S.E. i.u. = International Units ( $\mu \mathrm{mol}$ substrate hydrolysed $/ \mathrm{min} / \mathrm{litre}$ at $\left.37^{\circ} \mathrm{C}\right) . \Delta \mathrm{Tf}=$ Depression of freezing point. For other abbreviations, see the text.

* Values for intact animals are taken from Jones \& Glover (1973a).

ligation on the ductus deferens and histological sections of the caput epididymidis and testis recovered from vasectomized males at 2, 6 and 24 weeks did not reveal any back pressure effects on the testis or any disruption of spermatogenesis. This is in agreement with the findings of Paufler \& Foote (1969) who noted that there was an increase in the total number of spermatozoa in the cauda epididymidis after vasectomy but not in the caput region. It would appear, therefore, that during the first 24 weeks following vasectomy in the rabbit the accumulation of spermatozoa is accommodated entirely within the cauda epididymidis and the ductus deferens.

Despite considerable distention of the cauda epididymidis, the chemical composition of the luminal plasma was similar in all experimental groups, and, with one or two exceptions, showed few changes from values recorded for intact animals (Table 1). The concentration of inorganic ions decreased slightly after 6 weeks, but protein, osmotic pressure and the activity of the phosphatases and glycosidases all remained within normal limits. This is in contrast to the situation after castration when there is a rapid increase in the levels of sodium and 


\section{PLATE 1}

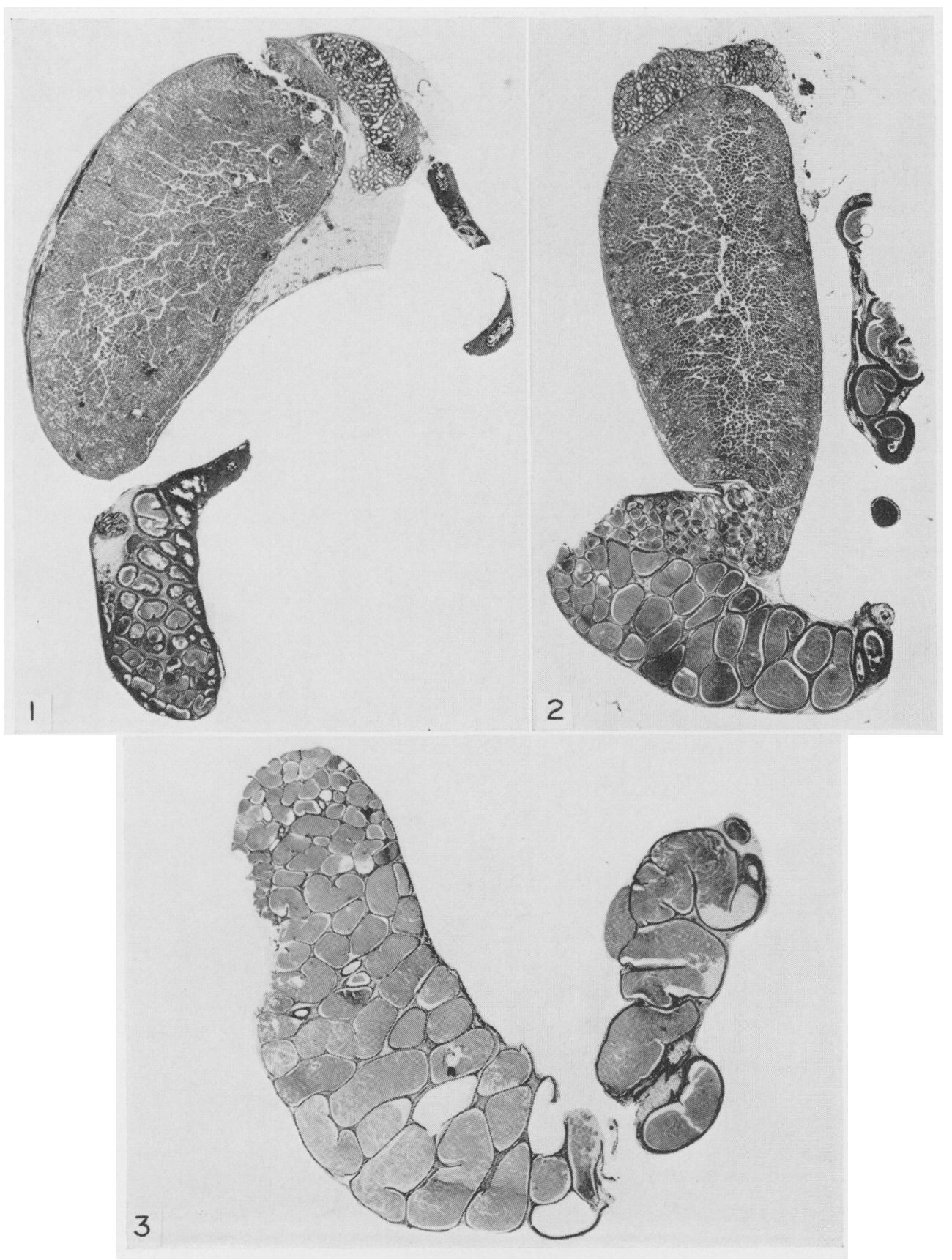

Staining technique: Harris's haematoxylin and cosin.

FIG. 1. Histological section of testis and cauda epididymidis from a control rabbit. $\times 2 \frac{1}{2}$. FIg. 2. Histological section of testis and cauda epididymidis 6 weeks after vasectomy. $\times 2 \frac{1}{2}$.

FIG. 3. Histological section of cauda epididymidis 24 wecks after vasectomy. $\times 2 \frac{1}{2}$. 
chloride ions in the plasma and a fall in protein concentration and enzyme activity (Jones \& Glover, 1973b). Jones (1973) has further postulated from this work that an increase in the levels of inorganic ions is indicative of reduced absorptive activity. In the present study, however, it was noticeable that, 12 and 24 weeks after vasectomy, the concentration of GPC fell to approximately half the normal levels whereas inorganic phosphate increased two- to threefold (Table 1). It is well known that GPG is a specific secretory product of the epididymis (Mann, 1964), and it may be that, although absorption was unaffected by vasectomy, there was some decline in secretory activity. An alternative explanation might be that there was a breakdown of GPC in the lumen. Jones (1973) has shown that after castration or artificial cryptorchidism there is a decrease in GPG in the plasma and that this is always accompanied by elevated phosphate levels. It is possible that the GPC is the source of the increased amount of phosphate under these circumstances but without further information on the simultaneous appearance of choline in the plasma it is difficult to reach a definite conclusion on this point.

At 6 and 24 weeks after vasectomy, all spermatozoa from the ductus deferens stained with eosin and $60 \%$ to $70 \%$ were decapitate, whereas from the proximal cauda epididymidis only $8 \%$ and $26 \%$, respectively, were eosinophilic and less than $18 \%$ were decapitate. This suggests that there was renewal and mixing of spermatozoa in the cauda epididymidis but that this may not have extended as far as the ductus deferens. These observations are of interest in relation to the increase in LDH activity after vasectomy (Table 1). Jones \& Glover (1973a, b) have shown that, when rabbit epididymal spermatozoa are damaged in vitro by cold-shock or degenerate in vivo in the epididymis, intracellular constituents leak into the surrounding plasma. In such circumstances, there is a correlation between the activity of $\mathrm{LDH}$ and the incidence of eosinophilic spermatozoa. Thus, it might be expected that there would be higher levels of LDH in plasma from the ductus deferens than from the cauda epididymidis. This may account for the rather wide variation in LDH levels between different animals since no attempt was made to collect plasma from different regions and it was always pooled. Further work is required to verify this point.

In conclusion, these results suggest that vasectomy does not seriously impair the capacity of the cauda epididymidis to maintain a stable 'milieu' in the lumen of the duct, and it seems unlikely that the normal maturation and survival of spermatozoa would be affected. It must be emphasized, however, that if a spermatocoele should develop in any region, then a different situation may arise and the function of the epididymis might then be impaired.

This work was financed by grants from the Ford Foundation and the Northern Ireland Ministry of Education. I am grateful to Mr H. S. Anger of the A.R.C. Unit of Reproductive Physiology and Biochemistry, Cambridge, for technical assistance with the histology.

\section{REFERENCES}

Amann, R. P. \& Almourst, J. O. (1962) Reproductive capacity of dairy bulls. VI. Effect of unilateral vasectomy and ejaculation frequency on sperm reserves; aspects of epididymal physiology. 7. Reprod. Fert. 3, 260. 
Gampeele, R. C., Dott, H. M. \& Glover, T. D. (1956) Nigrosin eosin as a stain for differentiating live and dead spermatozoa. F. agric. Sci., Camb. 48, 1.

Hooker, R. H. \& Gilmore, D. P. (1972) Obstruction of the vasa deferentia of the male rat and rabbit with silastic medical adhesive. Int. F. Fert. 17, 14.

IgBoeli, G. \& RaKha, A. U. (1970) Bull testicular and epididymal functions after long-term vasectomy. F. Anim. Sci. 31, 72.

Jones, R. (1973) Studies on the composition of epididymal plasma. Ph.D. thesis, University of Liverpool.

Jones, R. \& Glover, T. D. (1973a) The collection and composition of epididymal plasma from the cauda epididymidis of the rabbit. 7 . Reprod. Fert. 34, 395.

Jones, R. \& Glover, T. D. (1973b) The effects of castration on the composition of rabbit epididymal plasma. F. Reprod. Fert. 34, 405.

Laumas, K. R. \& Untyal, J. P. (1967) Disintegration of spermatozoa and testicular atrophy by a silastic block of vas deferens in rats. Indian F. exp. Biol. 5 (4), 199.

Macmillan, K. L., Desjardins, G., Kirton, K. T. \& Hafs, H. D. (1968) Gonadal and extragonadal sperm reserves after unilateral vasoligation in rabbits. Fert. Steril. 19, 982.

MANn, T. (1964) The biochemistry of semen and of the male reproductive tract. Methuen, London.

PAUfler, S. K. \& Foote, R. H. (1969) Sperm retention and resorption in sexually active rabbits with epididymal ligatures. Proc. Soc. exp. Biol. Med. 131, 1179.

Sacher, B. \& Schilling, E. (1973) Inhibition of spermatogenesis by vasectomy in the rabbit. Proc. VIIth Int. Congr. Anim. Reprod., Munich, Vol. III, p. 437. 